\title{
Volatile Analysis by Pyrolysis of Regolith for Planetary Resource Exploration
}

\author{
Daniel P. Glavin ${ }^{1 *}$, Charles Malespin ${ }^{1,2}$, Inge L. ten Kate ${ }^{3}$, Stephanie A. Getty ${ }^{1}$, Vincent E. Holmes ${ }^{1,4}$, Erik \\ Mumm $^{5}$, Heather B. Franz ${ }^{1,6}$, Marvin Noreiga ${ }^{1,7}$, Nick Dobson ${ }^{1,8}$, Adrian E. Southard ${ }^{1,2}$, Steven H. Feng ${ }^{1}$, Carl A. \\ Kotecki ${ }^{1}$, Jason P. Dworkin ${ }^{1}$, Timothy D. Swindle ${ }^{9}$, Jacob E. Bleacher ${ }^{1}$, James W. Rice', and Paul R. Mahaffy ${ }^{1}$
}

\begin{abstract}
1NASA Goddard Space Flight Center, 8800 Greenbelt Road, Greenbelt, MD 20771, USA, ${ }^{2}$ University Space Research Assocrates, 10211 Wincopin Circle, Columbia, MD 21044, USA; ${ }^{3}$ University of Oslo, Sem Sælands vei 24, NO-0316 Oslo, Norway; ${ }^{4}$ Bastion Technologies, Lanham, MD 20706, USA; ${ }^{5}$ Honeybee Robotics, 460 W. $34^{\text {th }}$ Street, New York, NY, 10001 , USA; ${ }^{6}$ Center for Research and Exploration in Space Science and Technology, 5523 Research Park Drive, University of Maryland Baltimore County, Baltimore MD 21228 , USA; ${ }^{7}$ ADNET Systems, Inc., 164 Rollins Ave., Rockville, MD 20852, USA; ${ }^{8}$ Northrup Grumman Corporation, 2980 Fairview Park Drive, Falls Church, VA 22042, USA; ${ }^{\circ}$ Lunar and Planetary Laboratory, University of Arizona, Tucson, AZ 85721, USA

*Corresponding author email: daniel.p.glavin@nasa.gov

charles.a.malespin@nasa.gov,science@ingeloes.com, stephanie.a.getty@nasa.gov, vincent.e.holmes@nasa.gov, mumm@honeybeerobotics.com, heather.b.franz@nasa.gov,marvin.g.noreiga@nasa.gov,nick.dobson@gmail.com, adrian.e.southard@nasa.gov, steven.h.feng@nasa.gov, carl.a.kotecki@nasa.gov,jason.p.dworkin@nasa.gov, tswindle@u.arizona.edu, jacob.e.bleacher@nasa.gov,james.w.rice@nasa.gov, and paul.r.mahaffy@nasa.gov
\end{abstract}

The extraction and identification of volatile resources that could be utilized by humans including water, oxygen, noble gases, and hydrocarbons on the Moon, Mars, and small planetary bodies will be critical for future long-term human exploration of these objects. Vacuum pyrolysis at elevated temperatures has been shown to be an efficient way to release volatiles trapped inside solid samples. In order to maximize the extraction of volatiles, including oxygen and noble gases from the breakdown of minerals, a pyrolysis temperature of $1400^{\circ} \mathrm{C}$ or higher is required, which greatly exceeds the maximum temperatures of current state-of-the-art flight pyrolysis instruments. Here we report on the recent optimization and field testing results of a high temperature pyrolysis oven and sample manipulation system coupled to a mass spectrometer instrument called Volatile Analysis by Pyrolysis of Regolith (VAPoR). VAPoR is capable of heating solid samples under vacuum to temperatures above $1300^{\circ} \mathrm{C}$ and determining the composition of volatiles released as a function of temperature.

Keywords - Evolved gas analysis, Desert Research And Technology Studies (DRATS), Lunar volatiles, Mass spectrometry, Planetary science, Resource utilization, Vacuum pyrolysis, Volatile Analysis by Pyrolysis of Regolith (VAPoR)

\section{INTRODUCTION}

Measuring the chemical composition of planetary bodies and their atmospheres is key to understanding the formation of the Solar System and the evolution of the planets and their moons. Moreover, in situ volatile measurements would enable a ground-truth assessment of the availability of resources such as water and oxygen, important for a sustained human presence on the Moon and beyond. Recent data from the Lunar CRater Observation and Sensing Satellite (LCROSS) revealed spectroscopic evidence for water-ice in the impact plume from Cabeus crater at a level corresponding to $\sim 6 \mathrm{wt} \%$ water-ice [1]. Several other volatiles including light hydrocarbons, sulfur bearing species, and carbon dioxide were also detected in the impact plume by LCROSS [1]. The detection of water-ice by LCROSS was consistent with previous observations by Clementine and the Lunar Prospector spacecraft showing that the lunar polar regions contain enhanced levels of hydrogen, a potential signature of water-ice [2]. One explanation for the presence of enhanced volatiles at the lunar poles is the delivery of water and other volatile species to the lunar surface by repeated cometary and asteroid impacts during the late heavy bombardment period $\sim 4.1-3.8$ Ga [3], followed by migration and concentration of the volatiles in permanently shadowed cold traps. Another possible source for some of the volatiles detected at the lunar poles, particularly hydrogen, is implantation by the solar wind. Any solar wind implanted hydrogen or volatiles in the surface regolith that migrate to the polar regions would be delayed from diffusing out of the permanently shadowed regolith by the extremely low temperatures [4].

Although the volatile content of lunar samples recovered from the equatorial regions of the Moon during the Apollo missions has been well studied $[5-8]$, to thoroughly characterize the volatile abundance, distribution, and isotopic composition of regolith at the lunar poles, in situ analyses by pyrolysis instruments with high resolution mass spectrometers will be required to fully quantify the presence and composition of the volatiles. To date, there have been no in situ volatile measurements on the Moon using pyrolysis mass spectrometry. The Lunar Atmosphere Composition Experiment (LACE) mass spectrometer on Apollo 17, did make surface volatile measurements of the tenuous lunar exosphere, but it was not equipped with pyrolysis ovens for evolved gas measurements of the regolith [9].

Vacuum pyrolysis is the most efficient way to release the 
widest range of volatiles from regolith samples, especially if the sample has been crushed prior to heating [10]. It has been shown previously in laboratory measurements that $90 \%$ of the volatile materials in Apollo samples can be released by pyrolysis stepped heating to $1400^{\circ} \mathrm{C}$ [6]. Many of the key volatiles of interest for planetary resource exploration can be extracted at temperatures of $1000^{\circ} \mathrm{C}$ or less (see Table 1). However, vacuum pyrolysis experiments of lunar simulants show that $\mathrm{O}_{2}$ can only be released at temperatures greater than $1200^{\circ} \mathrm{C}[11]$. In addition, the release of the noble gases $\mathrm{Ne}$, $\mathrm{Ar}, \mathrm{Kr}$, and $\mathrm{Xe}$ trapped in higher temperature mineral phases requires stepped heating to temperatures up to $1400^{\circ} \mathrm{C}[12$, 13]. Although other approaches have been used for the extraction of volatiles, including mechanical agitation [14] and laser heating [15], vacuum pyrolysis using a stable controlled heating ramp coupled with mass spectrometry will enable evolved gas measurements that can be used to determine the volatile distribution in a solid sample and provide important constraints on bulk chemistry and mineral composition.

\begin{tabular}{|c|c|c|}
\hline & VAPoR target gases & Temperature range $\left({ }^{\circ} \mathrm{C}\right)$ \\
\hline \multirow{5}{*}{$\begin{array}{l}\text { CHONS- } \\
\text { Inorganics }\end{array}$} & Atmospheric volatiles & Not applicable \\
\hline & $\mathrm{H}_{2} \mathrm{O}, \mathrm{H}_{2}, \mathrm{CO}_{2}, \mathrm{CO}, \mathrm{N}_{2}, \mathrm{SO}_{2}$ & $0-1400^{[6,7,17]}$ \\
\hline & ${ }^{13} \mathrm{Cl}^{12} \mathrm{C}$ ratio of $\mathrm{CO}_{2}$ & $100-1400^{[17]}$ \\
\hline & ${ }^{15} \mathrm{~N} /{ }^{14} \mathrm{~N}$ ratio in $\mathrm{N}_{2}$ & $600-1400^{[17]}$ \\
\hline & $\mathrm{HDO} / \mathrm{H}_{2} \mathrm{O}$ ratio & $0-1400$ \\
\hline \multirow{3}{*}{$\begin{array}{l}\text { Noble } \\
\text { Gases }\end{array}$} & $\mathrm{He}, \mathrm{Ne}, \mathrm{Ar}$ & $300-1400^{[17,18]}$ \\
\hline & $\begin{array}{l}\text { Isotope ratios }\left({ }^{3} \mathrm{He} /{ }^{4} \mathrm{He} \text {, }\right. \\
\left.{ }^{36} \mathrm{Ar} /{ }^{40} \mathrm{Ar}\right)\end{array}$ & $\begin{array}{l}\text { He: } 200-500^{[5]} \\
\text { Ar: } 300-1400^{[17,19]}\end{array}$ \\
\hline & $\begin{array}{l}{ }^{13} \mathrm{C} /{ }^{12} \mathrm{C} \text { ratio in } \mathrm{CO}_{2} \text { from } \\
\text { organics combustion }\end{array}$ & $400-500^{[18]}$ \\
\hline \multirow[t]{2}{*}{ Organics } & $\begin{array}{l}\text { Volatile hydrocarbons: } \\
\text { methane, ethane, benzene, } \\
\text { amines, alcohols, } \\
\text { formaldehyde }\end{array}$ & $300-1000^{[17]}$ \\
\hline & Water-ice in regolith & $0-100$ \\
\hline \multirow{4}{*}{$\begin{array}{l}\text { Other } \\
\text { Resources }\end{array}$} & $\mathrm{O}_{2}$ & $1100-1400^{[3]}$ \\
\hline & $\begin{array}{l}\text { Reduced inorganic gases } \\
\text { such as } \mathrm{HCN}, \mathrm{NH}_{3} \text {, and } \\
\mathrm{H}_{2} \mathrm{~S}\end{array}$ & $\begin{array}{l}\mathrm{HCN} / \mathrm{NH}_{3}: 100-900^{[17]} \\
\mathrm{H}_{2} \mathrm{~S}: 700-1300^{[6]}\end{array}$ \\
\hline & ${ }^{3} \mathrm{He}$ relative abundance & $\mathrm{He}: 200-500^{[5]}$ \\
\hline & ${ }^{3} \mathrm{He}$ relative abundance & He: $200-500^{[5]}$ \\
\hline
\end{tabular}

Several previous and current flight instruments used the pyrolysis approach to extract volatiles from solid regolith (summarized in Table 2). The Viking landers each carried a gas chromatography mass spectrometry (GCMS) instrument equipped with three ovens capable of heating regolith samples at different temperatures up to $500^{\circ} \mathrm{C}[20,21]$. More recently, the Thermal and Evolved Gas Analyzer (TEGA) instrument on the Phoenix Mars lander heated polar regolith samples up to $950^{\circ} \mathrm{C}$ and analyzed the evolved gases using a mass spectrometer [22]. The Rosetta mission, currently on its way to comet $67 \mathrm{P} /$ Churyumov-Gerasimenko, includes two evolved gas mass spectrometer instuments, the Cometary Sampling and Composition (COSAC) experiment [23] and Ptolemy [24]. These instruments contain very small pyrolysis ovens, capable of heating samples to $600^{\circ} \mathrm{C}$ and $800^{\circ} \mathrm{C}$, respectively. Most recently, the Sample Analysis at Mars (SAM) instrument suite on the Mars Science Laboratory (MSL) mission that is scheduled to launch in 2011 contains two separate pyrolysis ovens, each capable of being heated up to $950-1100^{\circ} \mathrm{C}$ for volatile analysis of solid samples by gas chromatography mass spectrometry and tunable laser spectroscopy [25]. None of these current flight ovens are designed to heat solid samples to temperatures above $1100^{\circ} \mathrm{C}$ as required to release the full range of volatiles from planetary samples (Tables 1 and 2).

Future robotic missions to remote planetary surfaces will likely be highly resource constrained and will require smaller and simpler instrumentation than their predecessors. Therefore, the development of lower mass and power evolved gas mass spectrometer instruments that maintain or exceed the performance of previous instruments will be a key challenge for upcoming missions. The Volatile Analysis by Pyrolysis of Regolith (VAPoR) instrument is a simplified version of its predecessor SAM, and enables compositional and isotopic measurements of volatiles in planetary atmospheres and exospheres and released from solid surface samples using pyrolysis mass spectrometry on airless bodies including the Moon, asteroids and comets, and the icy moons of the Outer Planets [16]. With the addition of a miniature turbo pump, VAPoR could also operate in higher pressure planetary environments including Mars and Titan.

\begin{tabular}{|c|c|c|c|}
\hline & Viking $^{|21|}$ & TEGA $^{|22|}$ & $\operatorname{Cos} A^{[23]}$ \\
\hline $\begin{array}{l}\text { Temperature } \\
\text { range }\end{array}$ & $\begin{array}{l}50,200,350 \text {, } \\
\text { and } 500^{\circ} \mathrm{C} \text {. }\end{array}$ & Ambt. to $950^{\circ} \mathrm{C}$ & Ambt, to $600^{\circ} \mathrm{C}$ \\
\hline $\begin{array}{l}\text { Oven } \\
\text { materials }\end{array}$ & Ceramic. & $\begin{array}{l}\text { Nickel. } \\
\text { Platinum resistance } \\
\text { wire. } \\
\text { Ceramic coating. }\end{array}$ & $\begin{array}{l}\text { Platinum. } \\
\text { Platinum } \\
\text { resistance wire. } \\
\text { Glass. }\end{array}$ \\
\hline $\begin{array}{l}\text { Oven/cup size } \\
\text { diameter } \\
\text { length } \\
\text { volume }\end{array}$ & $\begin{array}{l}2 \mathrm{~mm} \\
19 \mathrm{~mm}^{3} \\
60 \mathrm{~mm}^{3}\end{array}$ & $\begin{array}{l}7.2 \mathrm{~mm} \\
21.6 \mathrm{~mm} \\
38 \mathrm{~mm}^{3} \text { (sample) }\end{array}$ & $\begin{array}{l}3 \mathrm{~mm} \\
6 \mathrm{~mm} \\
42 \mathrm{~mm}^{3}\end{array}$ \\
\hline & Ptolemy ${ }^{|24|}$ & $\mathbf{S A M}^{[25]}$ & VAPoR \\
\hline $\begin{array}{l}\text { Temperature } \\
\text { range } \\
\text { Oven } \\
\text { material }\end{array}$ & $\begin{array}{l}\text { Ambt. to } \\
800^{\circ} \mathrm{C} \\
\text { Platinum. } \\
\text { Platinum } \\
\text { resistance } \\
\text { wire. } \\
\text { Glass. }\end{array}$ & $\begin{array}{l}\text { Ambt. to } \sim 1000^{\circ} \mathrm{C} \\
\text { Inconel } 693 \text { tube } \\
\text { surrounded by } \\
\text { alumina sleave. } \\
\text { ZGS Platinum-10\% } \\
\text { Rhodium resistance } \\
\text { wire. }\end{array}$ & $\begin{array}{l}\text { Ambt. to } \\
1400^{\circ} \mathrm{C} \\
\text { Alumina } \\
\text { crucible. } \\
\text { ZGS Platinum- } \\
10 \% \text { Rhodium } \\
\text { resistance wire. }\end{array}$ \\
\hline $\begin{array}{l}\text { Oven/cup size } \\
\text { diameter } \\
\text { length } \\
\text { volume }\end{array}$ & $\begin{array}{l}3 \mathrm{~mm} \\
6 \mathrm{~mm} \\
42 \mathrm{~mm}^{3}\end{array}$ & $\begin{array}{l}7 \mathrm{~mm} \mathrm{I.D.} \mathrm{(qtz.} \mathrm{cup)} \\
20.5 \mathrm{~mm} \text { (qtz. cup) } \\
789 \mathrm{~mm}^{3} \text { (qtz. cup) }\end{array}$ & $\begin{array}{l}6.2 \mathrm{~mm} \mathrm{I.D.} \\
18.2 \mathrm{~mm}^{3} \\
539 \mathrm{~mm}^{3}\end{array}$ \\
\hline $\begin{array}{l}\text { Number of } \\
\text { ovens/cups }\end{array}$ & 3 & $\begin{array}{l}2 \text { ovens, } 74 \text { sample } \\
\text { cups (qtz./inconel) }\end{array}$ & 6 \\
\hline
\end{tabular}

The preliminary VAPoR flight instrument concept (Fig. 1) combines a sample carousel of up to six individually heated pyrolysis ovens with a reflectron time of flight mass spectrometer [26]. The VAPoR gas processing system includes two gas manifolds, heated transfer lines, and two separate gas reservoirs for calibration of the mass 
spectrometer and oxygen for combustion experiments. Powdered rock or soil samples collected from a rover or lander drill or scoop and delivered through the solid sample tube to one of the VAPoR ovens can then be heated by a controlled ramp from ambient to temperatures up to $1400^{\circ} \mathrm{C}$ to release the volatile constituents for direct measurement by the mass spectrometer. Two independent units have been built and tested to understand the performance of the different instrument components. A laboratory breadboard was developed to test, optimize, and calibrate the reflectron time of flight mass spectrometer (TOF-MS) component of VAPoR inside a separate vacuum chamber and is discussed in more detail in Section V and elsewhere [26]. A separate portable field unit consisting of a custom made pyrolysis oven coupled to a commercial RGA quadrupole mass spectrometer, vacuum manifold and turbomolecular pumping station, was built to demonstrate the feasibility of conducting vacuum pyrolysis evolved gas measurements in the field and has been discussed previously [16].

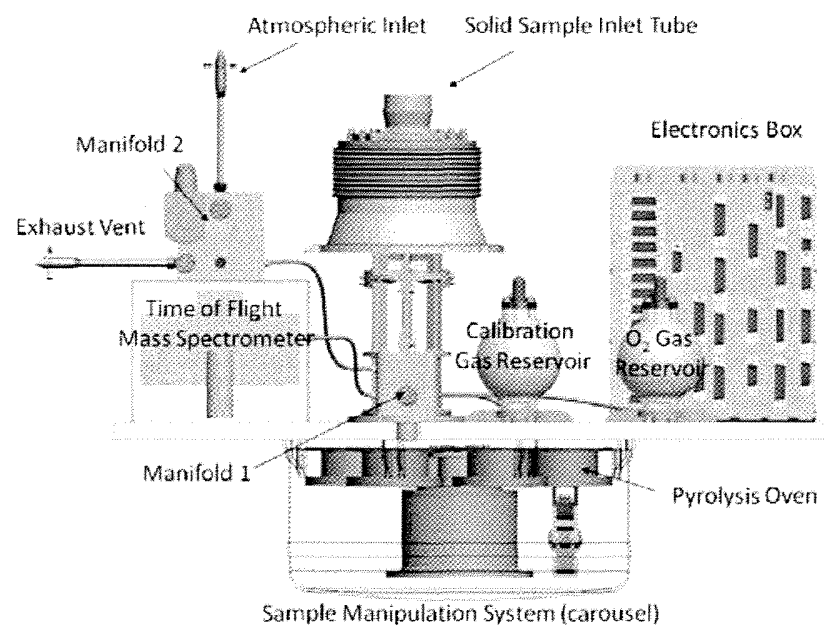

Fig. 1. Cross sectional view of the preliminary VAPoR flight instrument concept which combines a sample carousel containing six separate pyrolysis ovens integrated to a reflectron time of flight mass spectrometer for volatile analyses on the surface of planetary bodies throughout the Solar System.

The focus of this paper will be on the high temperature pyrolysis oven and six-position oven carousel components that were recently integrated to the VAPoR field instrument (Fig. 2). Here we will describe the VAPoR instrument and key science measurement objectives enabled by high temperature pyrolysis, provide details on the new oven and carousel designs, and present recent experimental results obtained during NASA's 2011 Desert Research And Technology Studies (DRATS) field campaign at Black Point Lava Flow in Arizona.

\section{VAPOR INSTRUMENT AND ANALYTICAL PROTOCOL}

\section{A. Science Measurement Objectives}

There are at least four key planetary science and resource exploration measurement objectives that can be achieved using VAPoR: (1) confirm the abundances of water detected on the Moon by LCROSS [1] and discriminate between adsorbed water, water-ice, and water released from hydrated minerals; (2) measure the distribution and isotopic (D/H ratio) composition of water and the presence of other volatiles including hydrocarbons to establish their origin(s), (3) measure the total abundances of oxygen released by breakdown of silicate minerals during high temperature pyrolysis for large-scale in situ resource utilization technology development, and (4) contribute to in situ geochronology by thermal extraction of argon and ${ }^{40} \mathrm{Ar}$ measurements needed for $\mathrm{K}-\mathrm{Ar}$ radiometric age dating [27].

VAPoR will focus on the analyses of $\mathrm{C}, \mathrm{H}, \mathrm{O}, \mathrm{N}$, and $\mathrm{S}$ containing volatiles commonly released from minerals, including $\mathrm{H}_{2} \mathrm{O}, \mathrm{CO}_{2}, \mathrm{CO} / \mathrm{N}_{2}$, and $\mathrm{SO}_{2}$. The pyrolysis temperature profile was optimized for the detection of simple aliphatic and aromatic hydrocarbons such as methane, ethane, benzene, and alkylbenzene. In addition, VAPoR will measure the distribution and isotopic composition of the noble gases $\mathrm{He}, \mathrm{Ne}$, and Ar. These measurements are important for understanding the contribution of noble gases from solar wind implantation and cosmic ray bombardment and potentially even outgassing from the interior of a planetary body. Measurements of ${ }^{40} \mathrm{Ar}$ extracted from rock samples by VAPoR high temperature pyrolysis is also important for $\mathrm{K}$-Ar age dating if $\mathrm{K}$ abundances can be derived by another measurement technique. An overview of the target volatiles of VAPoR and their release temperatures is given in Table 1.

\section{B. VAPoR Field Instrument Description}

The VAPoR field instrument tested during DRATS shown in Figure 2 consists of a six-port stainless steel vacuum manifold (MDC Vacuum Products) connected to a six-position sample manipulation system (SMS, Honeybee Robotics) containing two custom made high temperature pyrolysis ovens developed at NASA Goddard Space Flight Center (GSFC).

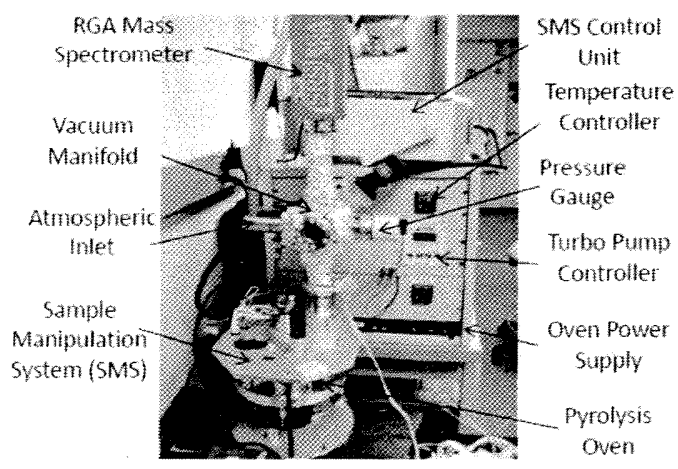

Fig. 2. The VAPoR field instrument, which includes a new sample manipulation system and high temperature pyrolysis oven for evolved gas analysis of powdered solid samples.

The vacuum manifold is also connected to an atmospheric gas inlet (MDC precision leak valve), a cold cathode ion pressure gauge (Pfeiffer Vacuum PKR 251), and a residual gas analyzer (Stanford Research Systems, RGA 300, mass range: 1-300 amu), and is actively pumped using a turbomolecular pumping system (Pfeiffer Vacuum TSU071E, TC600), A $30 \mathrm{~V}$ power supply (Sorensen DLM 20-30) is used to power the 
ovens and a separate control unit (Honeybee Robotics) is used to power and command the SMS. Two Watlow EZ Zone temperature controllers are used to control the pyrolysis oven ramp rate (set at $20^{\circ} \mathrm{C} / \mathrm{min}$ ) and manifold heater temperature (set at $50^{\circ} \mathrm{C}$ ). A ruggedized laptop computer (Panasonic Toughbook) with a custom Labview platform is used to control the RGA and collect mass spectrometer, oven voltage and current, and oven temperature data obtained from the instrument. The instrument is set up to enable direct line of sight from the heated sample in an oven to the ionization region of the RGA mounted at the top of the manifold. The RGA was setup to scan over the mass range 1-100 amu during the pyrolysis heating experiment. The current VAPoR field instrument does not include the TOF-MS, which is being tested and optimized separately in a larger vacuum chamber at NASA GSFC (Section V).

\section{Solid Sample Analysis Protocol}

A sample collection and analytical protocol was developed for the VAPoR field instrument prior to NASA's 2011 DRATS field test. The mortar and pestle, stainless steel spatulas, glass vials, quartz sample holders and glass wool used in sample preparation and storage were all baked at $500^{\circ} \mathrm{C}$ in air for $3 \mathrm{~h}$. Rock and soil samples were collected by crewmembers in the field using clean metal tongs or shovels. Samples were subsequently wrapped in ultrahigh vacuum foil (All-Foils, Inc.) prior to bagging inside polyethylene bags (Whirl-Pak) in order to minimize hydrocarbon contamination of the samples during the collection process. This approach eliminated direct contact between the sample and the bags or gloves of the crewmembers, as commonly occurs during the standard sample collection procedure used during DRATS [28]. Photodocumentation and preliminary microscopic imaging analyses of the samples were conducted inside the DRATS Geolab [29], which is a glovebox located inside NASA's Habitat Demonstration Unit that is designed for scientific analyses of samples collected during a mission. During the field test VAPoR was a standalone instrument physically located outside of the Geolab and subsequent to the standard crew analyses a small fragment of each sample was chipped off for VAPoR evolved gas measurements. One of the Geolab samples studied by VAPoR (sample 0212, Fig. 3) was a vesicular basalt containing up to $3 \mathrm{~mm}$ diameter vesicles. Results from the evolved gas analysis of a small fragment of this sample is discussed in Section IV.

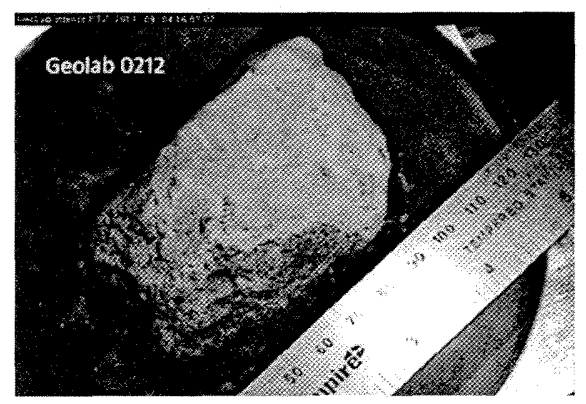

Fig. 3. Image of rock sample 0212 processed in the Geolab and analyzed by VAPoR. Sample 0212 is a vesicular basalt fragment covered with light brown soil with a total mass of $156 \mathrm{~g}$ (photo credit: NASA).
Prior to VAPoR analysis, each sample fragment was crushed using a ceramic mortar and pestle and the resulting powder passed through a stainless steel metal sieve (ASTM No. $100,<150 \mu \mathrm{m}$ ). The sieved powder was transferred into a clean $8 \mathrm{ml}$ screw capped glass vial using a stainless steel spatula. Since many rock samples will melt inside the oven crucible when heated to temperatures above $1200^{\circ} \mathrm{C}$, the rock powders $(\sim 10 \mathrm{mg})$ were each loaded inside a separate quartz tube sample holder ( $3 \mathrm{~mm} \mathrm{ID,} 5 \mathrm{~mm} \mathrm{OD,} 25 \mathrm{~mm}$ length) packed with quartz glass wool at the bottom, and the entire quartz holder was inserted into the VAPoR oven (see Fig. 4).

Although the VAPoR pyrolysis oven can handle much larger sample sizes (up to $\sim 1$ gram), we have found that at elevated temperatures, samples with masses over $10 \mathrm{mg}$ can lead to high manifold pressures $\left(>10^{-4} \mathrm{mbar}\right.$ ) during pyrolysis, which prohibits the use of the mass spectrometer during the evolved gas measurements. A $<150 \mu \mathrm{m}$ fused silica powder (120/P, Precison Electro Minerals Co., Inc.) that had been baked in air for $6 \mathrm{hr}$ at $900^{\circ} \mathrm{C}$ was used as a procedural blank to characterize the background of volatiles in the VAPoR system during a solid sample pyrolysis experiment. The fused silica powder was also used in the solid sample temperature calibration experiments to determine the relationship between the oven ramp tamperature and the actual temperature of a sample inside the quartz tube holder (see Section IIIB).

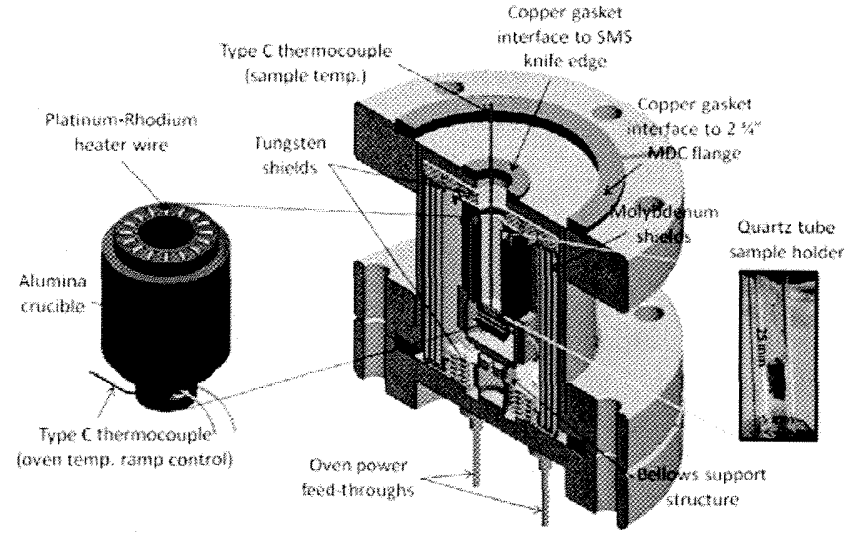

Fig. 4. Diagram of the main components of the high temperature pyrolysis oven custom made for the VAPoR field instrument. Solid samples are placed inside quartz tube sample holders (right inset), which are then inserted directly into the alumina oven crucible for evolved gas analysis. For the flight instrument, solid samples would be dropped by an astronaut or robotic sample acquisition system through an inlet tube directly into the alumina crucible.

After each quartz sample tube is loaded with a sample, the SMS is rotated into the sample receive position that places the oven directly beneath the solid sample inlet tube port. The quartz tube is then dropped inside the alumina crucible oven as illustrated in Figure 4. The oven is then rotated by the SMS into the pyrolysis position directly under a knife-edge interface to the vacuum manifold (additional details on SMS in Section IIID). The pyrolysis oven is raised up to engage the SMS knife-edge interface and create a vacuum tight knifeedge seal on the inner copper gasket on the top flange of the oven. After sealing, the turbo pump is started and allowed to spin up to $1500 \mathrm{~Hz}$. The vacuum manifold is pumped down to a pressure of $\sim 10^{-7}$ to $10^{-8}$ mbar prior to pyrolysis oven 
heating. In this pressure range, the RGA is turned on and the filament left on for 15 minutes to warm up. The oven power leads are connected from the power feedthroughs on the base of the oven directly to the front panel of the power supply and the oven set to an initial temperature of $50^{\circ} \mathrm{C}$.

For each EGA measurement, the pyrolysis oven crucible was heated at a controlled rate of $20^{\circ} \mathrm{C} / \mathrm{min}$ from $50^{\circ} \mathrm{C}$ up to $1000^{\circ} \mathrm{C}$ using the Watlow temperature controller based on type $\mathrm{C}$ thermocouple temperature measurements of the bottom of the alumina crucible (Fig. 4 left). Previous evolved gas measurements have shown that ramp rates of $20^{\circ} \mathrm{C} / \mathrm{min}$ or less are desired for sufficient time separation of volatiles released from the sample required for improved volatile mass identification. It should be noted that at $1000^{\circ} \mathrm{C}$, the actual temperature inside the oven that the sample is heated to is much higher than the measured oven temperature based on direct type $\mathrm{C}$ thermocouple measurements of the inside of the quartz sample holder made in separate experiments (discussed in Section IIIB). The vacuum manifold was kept at $50^{\circ} \mathrm{C}$ through the entire pyrolysis experiment to keep water and other volatiles from condensing on the internal surfaces of the manifold

While ramping the oven, analyses of the volatiles released from the sample were made by the RGA quadrupole mass spectrometer by repeated unit mass scans (mass range 2 to $100 \mathrm{Da}$ ) throughout the pyrolysis experiment. The sensitivity of the faraday cup collector used in the RGA is $2 \mathrm{x}$ $10^{-4} \mathrm{~A} /$ Torr and the measured current for each mass/charge $(\mathrm{m} / \mathrm{z})$ ratio is converted to a partial pressure (Torr) and stored in LabView. Each individual mass can then be plotted separately as a function of oven temperature in LabView to obtain an evolved gas plot. In addition to the primary volatile components typically released from terrestrial samples (water, carbon dioxide, sulfur dioxide, and nitrogen/carbon monoxide), we are also interested in identifying characteristic mass fragments of simple aliphatic (e.g., $\mathrm{m} / \mathrm{z} 27,43,55,57$ ) and aromatic (e.g., $\mathrm{m} / \mathrm{z} 78,91$ ) hydrocarbons released from the samples. The unit mass resolution of the RGA was not sufficient to make isotopic $\mathrm{C}, \mathrm{N}$, and $\mathrm{H}$ isotope measurements of the volatiles released; however future integration of a higher mass resolution TOF-MS to the VAPoR pyrolysis oven should greatly improve current capability.

\section{PYROLYSIS OVEN AND CAROUSEL MECHANISM}

\section{A. Oven Design}

The VAPoR pyrolysis oven (Fig. 4) was designed to bring the sample to a maximum temperature of $1400^{\circ} \mathrm{C}$ to release volatiles for direct mass spectrometer analysis. In contrast to the SAM instrument ovens [25], where quartz or metal cups containing the solid sample are inserted into an inconel tube surrounded by a platinum wire threaded alumina insulator, the VAPoR alumina oven crucible is the cup. This enables more efficient and direct heating of a solid sample placed at the bottom of the crucible. Like SAM, the VAPoR oven uses a zirconium grain strengthened (ZGS) platinum-rhodium alloy wire $(0.51 \mathrm{~mm})$ as the heater. This wire is threaded up and down through small channels in a custom-made alumina crucible (Technology Assessment and Transfer, Inc.) with an internal volume of $539 \mathrm{~mm}^{3}$. The VAPoR alumina crucibles are designed to hold up to $\sim 1$ gram of sample powder (assuming a density of $2 \mathrm{~g} / \mathrm{cm}^{3}$ ). As discussed previously, VAPoR requires only a very small volume of sample $(\sim 5$ $\mathrm{mm}^{3}$ or $\sim 10 \mathrm{mg}$ ) for each evolved gas analysis, which means that each oven can be refilled multiple times for over 100 evolved gas analyses before the crucible volume is completely full. However, it should be noted that the power required to heat a full oven would be substantially higher than in an oven containing a single $5 \mathrm{~mm}^{3}$ sample volume aliquot.

In order to minimize the power required to heat a sample, a set of four evenly spaced rhenium-coated precisionfabricated molybdenum vertical radiation shields surround the alumina crucible. In addition, a stack of ten custom made tungsten shields located below and above the alumina crucible are also used to improve the thermal design of the oven. To minimize the heat loss through the bottom of the crucible, an inconel bellows support structure is used. The entire oven assembly is housed inside a standard 2.75 in. stainless steel 304 flange and nipple assembly so that the oven can be mounted and sealed directly to the VAPoR vacuum manifold via a copper gasket and knife-edge. The bottom flange of the oven was modified to include a set of two copper power feedthroughs as well as two type $\mathrm{C}$ thermocouple feedthroughs (not seen in Fig. 4 cross-section). The platinumrhodium heater wire is connected directly to the power feedthroughs and a type $C$ thermocouple wire $(0.25 \mathrm{~mm}$ dia. $)$ connects from the thermocouple feedthroughs directly to the base of the oven directly below the quartz sample tube. A separate type $\mathrm{C}$ thermocouple was placed at the bottom of the quartz tube and connected to type $\mathrm{C}$ thermocouple feedthroughs in a separate flange mounted directly above the oven at the top of the vacuum manifold. The thermocouple inside the sample was only used during the sample temperature calibration experiment and was removed during actual pyrolysis EGA measurements given concern that some of our samples would melt around the thermocouple at temperatures above $1200^{\circ} \mathrm{C}$. In SAM, the ovens do not use thermocouples to determine the temperature of the ovens during pyrolysis heating, but instead the oven temperature is derived from the resistance of a second platinum heater wire threaded through the alumina insulator.

Based on SAM pyrolysis oven testing, and the fact that the volume heated by the VAPoR oven is smaller than in the SAM ovens (Table 2), we expect that the VAPoR oven would achieve maximum sample temperatures of $1400^{\circ} \mathrm{C}$ with a power consumption that is less than the equivalent power consumption of the SAM flight ovens at the same temperature $\left(\sim 36 \mathrm{~W}\right.$ at $950^{\circ} \mathrm{C}$ for SAM). Furthermore, in the SAM oven design the temperature of a sample inside a cup is $\sim 50-100^{\circ} \mathrm{C}$ lower than the external platinum wire temperature as determined by thermocouple measurements of the interior of the quartz cup. Therefore with the SAM ovens, the power required to get the sample to $950^{\circ} \mathrm{C}$ would likely be higher than $36 \mathrm{~W}$.

\section{B. Solid Sample Temperature Calibration}

In order to determine the relationship between the oven temperature measured from the bottom of the crucible and the actual temperature that a powdered sample reaches inside the quartz sample holder, we mounted a second type $\mathrm{C}$ 
thermocouple to the top of the VAPoR vacuum manifold cross so that the end of the thermocouple was located directly inside the quartz sample holder filled with $10 \mathrm{mg}$ of fused silica at the bottom of the oven crucible (Fig. 4). The oven crucible was then heated at a controlled ramp rate of $20^{\circ} \mathrm{C} / \mathrm{min}$ from ambient temperature up to $1000^{\circ} \mathrm{C}$ as measured by the thermocouple located at the bottom of the crucible. During the heating ramp we measured the oven crucible temperature, the temperature inside the quartz tube with and without fused silica, and the oven current and voltage. A plot of the oven crucible temperature compared to the actual sample temperature inside the quartz tube holder is shown below (Fig. 5).

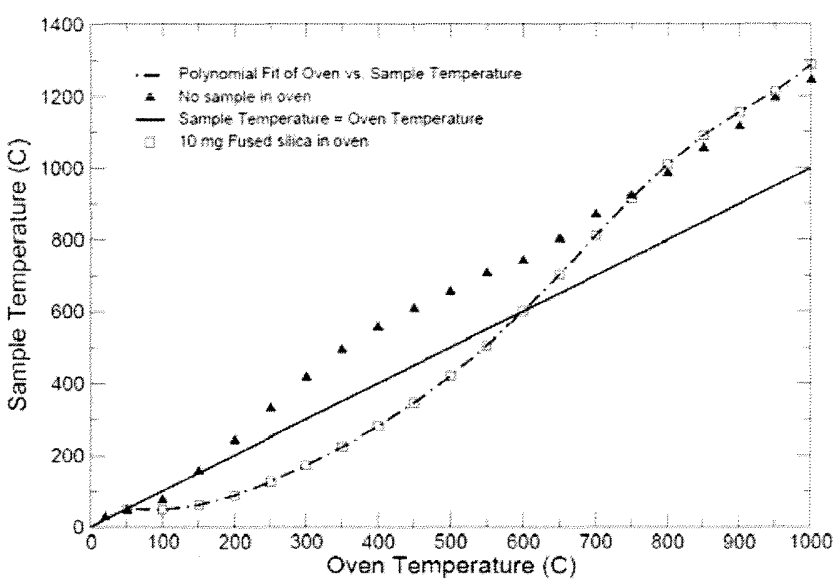

Fig. 5. Plot showing sample temperature $\left({ }^{\circ} \mathrm{C}\right)$ vs. alumina oven temperature $\left({ }^{\circ} \mathrm{C}\right)$ as measured with separate type $\mathrm{C}$ thermocouples during vacuum pyrolysis of an empty quartz tube and quartz sample tube containing $10 \mathrm{mg}$ of fused silica powder after heating the oven at a pressure of $\sim 10^{-7} \mathrm{mbar}$ to $1000^{\circ} \mathrm{C}$ at a rate of $20^{\circ} \mathrm{C} / \mathrm{min}$. The solid line represents the case where the sample temperature inside the quartz tube is identical to the temperature of the bottom of the alumina crucible. The dashed line is the polynomial best fit of the temperature of the fused silica sample as a function of oven temperature.

The thermocouple data show that when there was no solid sample inside an empty quartz holder, the temperature inside the quartz tube was significantly higher $\left(\sim 100-200^{\circ} \mathrm{C}\right)$ at temperatures above $200^{\circ} \mathrm{C}$ than the temperature measured at the bottom of the alumina crucible. This is likely due to the fact that the platinum-rhodium heater wires are wrapped predominately around the sides of the alumina crucible and most of the oven-generated heat is reflected directly to the center of the crucible by the vertical and horizontal shielding (Fig. 4). We also observed a significant sample temperature lag of $\sim 100^{\circ} \mathrm{C}$ at lower temperatures compared to the oven crucible temperature when $10 \mathrm{mg}$ of fused silica powder was placed inside the quartz sample holder. A much slower ramp rate than the $20^{\circ} \mathrm{C} / \mathrm{min}$ ramp rate used in these experiments would be required to reduce this measured sample temperature lag. At $\sim 600^{\circ} \mathrm{C}$ the sample temperature and oven temperature reached equilibrium; however, above $600^{\circ} \mathrm{C}$ the sample temperature increased rapidly up to a temperature of $\sim 1300^{\circ} \mathrm{C}$ (Fig. 5), greatly exceeding the temperature measured at the bottom of the crucible $\left(1000^{\circ} \mathrm{C}\right)$. Although the platinumrhodium heater wire used in the crucible could be heated to higher temperatures (up to $1400^{\circ} \mathrm{C}$ ), we stopped the experiment at a sample temperature of $\sim 1300^{\circ} \mathrm{C}$ to ensure that we did not melt the quartz sample tube and risk damage to the alumina crucible.

Since we did not measure sample temperature directly during an actual evolved gas analysis run (many rock samples will melt at $1300^{\circ} \mathrm{C}$ and could destroy the thermocouple), the fused silica sample temperature data was fit (Fig. 5 dashed line) to a polynomial function. Through a comparison of the polynomial fit to repeated actual sample temperature measurements, we have found that the sample temperature can be calculated directly from the measured oven crucible temperature with an accuracy of $\pm 5^{\circ} \mathrm{C}$ over the entire temperature range. It should be noted that the relationship between sample temperature and oven temperature was determined for a $10 \mathrm{mg}$ sized fused silica sample and samples with a different mineral composition or with larger masses might not follow the same polynomial fit.

\section{Oven Power Data}

In order to determine the power required to heat a solid sample, the oven current and voltage were measured as a function of oven temperature during each pyrolysis EGA run. The oven temperature was then converted to sample temperature based on a polynomial fit and the sample temperature then plotted vs. oven power (W). In Figure 6, plots of the oven power vs. calculated sample temperature are shown for the empty quartz holder and the quartz tube containing $10 \mathrm{mg}$ of fused silica. We found that a peak power of $64 \mathrm{~W}$ was required to heat the inside of the quartz tube up to a temperature of $1300^{\circ} \mathrm{C}$ and $\sim 60 \mathrm{~W}$ continuous power $(15$ $\mathrm{V}, 4 \mathrm{~A}$ ) was required to maintain that temperature. A slightly higher power ( $\sim 70 \mathrm{~W}$ peak) was required to heat up the fused silica powder to the same temperature as the empty quartz tube, which is probably due to a higher mass in the oven crucible.

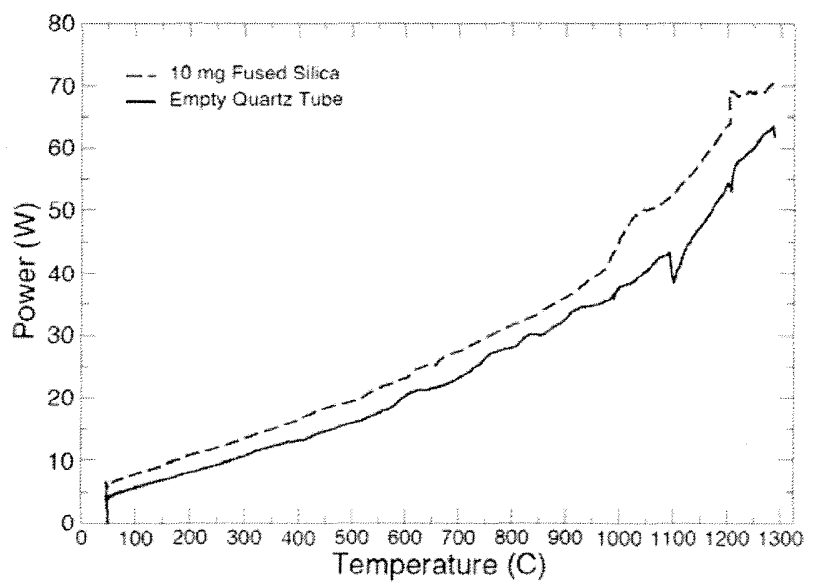

Fig. 6. Plot showing the peak power consumption (W) as a function of calculated sample temperature $\left({ }^{\circ} \mathrm{C}\right)$ of the alumina oven containing an empty quartz tube and a quartz tube filled with $10 \mathrm{mg}$ of fused silica powder. The oven was heated at $\sim 10^{-7}$ mbar to $1000^{\circ} \mathrm{C}$ at a rate of $20^{\circ} \mathrm{C} / \mathrm{min}$.

To reach a temperature of $950^{\circ} \mathrm{C}$, only $33 \mathrm{~W}$ of power was required for the VAPoR oven, which is less power than required by SAM pyrolysis at the same temperature. Based upon the power trends measured for the VAPoR oven (Fig. 6), we estimate that $\sim 75-80 \mathrm{~W}$ of power would be required to heat 
a $10 \mathrm{mg}$ solid sample to $1400^{\circ} \mathrm{C}$, although less continuous power would be required to maintain that temperature. Further optimization of the VAPoR oven design including the addition of alumina insulating material at the base of the Inconel 693 bellows support structure could help reduce heat loss from the base of the oven.

\section{Oven Carousel Design}

The sample manipulation system (SMS) developed for VAPoR is a two degree of freedom robotic system capable of accommodating up to six independent pyrolysis ovens (Fig. 7). A similar SMS concept was developed for the SAM experiment on the MSL mission which contains a total of 74 sample cups that can also accept solid sample through a solid sample inlet tube (SSIT) and then be raised individually inside one of two stationary pyrolysis ovens and sealed inside the oven using a knife-edge against a copper gasket on the cup stem. Unlike SAM, in the VAPoR SMS design the entire pyrolysis oven is rotated and sealed directly to the mass spectrometer vacuum manifold. One degree of freedom rotates the carousel to position a selected pyrolysis oven directly beneath the mass spectrometer vacuum manifold or the SSIT. The second degree of freedom raises and lowers the carousel as well as the cleats which preload an annealed copper gasket brazed to the top flange of the oven into a 1055 medium carbon steel knife-edge seal at the manifold location. The seal force has an accuracy of $\pm 10 \%$ and was designed to provide repeated leak tight $\left(<1 \times 10^{-5} \mathrm{cc} \mathrm{He} / \mathrm{sec}\right)$ seals at the oven manifold interface. Both degrees of freedom are actuated via the same gearmotor. A toggle/clutch mechanism dictates whether the gearmotor rotates or elevates the carousel.

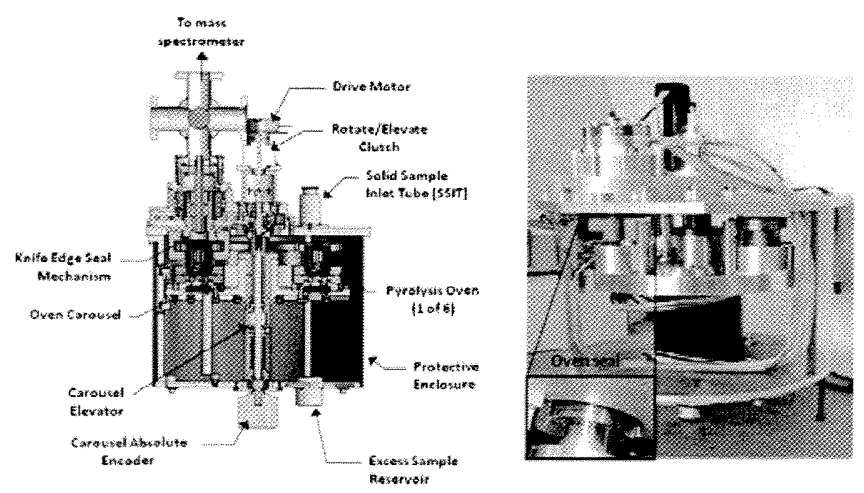

Fig. 7. A cross sectional view of the VAPoR SMS mechanical design (left) and fully assembled SMS hardware containing one pyrolysis oven (right) prior to integration to the field instrument.

Although a solid sample inlet tube was included in the VAPoR flight instrument concept, this component was not integrated to the VAPoR SMS. The sample reservoir shown in the mechanical design (Fig. 7, left) can be utilized as a "trash-can" for excess sample inside the SSIT, but is not included in the current SMS hardware. The SMS also includes a plastic enclosure to protect the moving parts within the SMS from dust contamination. The SMS control unit (Fig. 2) provides a graphical user interface to issue commands to the SMS and monitor the system status. Fully autonomous software routines allow the user to select between a stow, a detach, and a pyrolysis sequence. The control system also has the capability to direct power to a selected pyrolysis oven. Thus, all pyrolysis oven electrical connections (power and thermocouple leads) are self contained inside the SMS and the control system ensures that only the oven selected for an experiment is powered by the VAPoR power supply.

The stow routine preloads the carousel and the ovens into the primary plate in the center of the SMS mechanism. The purpose of the stow routine is to secure the system for transport (field unit). This routine can also be used for a flight instrument to secure the SMS and the pyrolysis ovens for launch and roving operations during surface operations of a landed mission. The pyrolysis routine sequences the system through the steps necessary to load the sample into an oven and initiate the pyrolysis heating sequence for an evolved gas measurement. The routine first places the specified oven at the sample inlet so that the sample quartz tube can be inserted into the pyrolysis oven crucible. When the sample has been delivered to the oven, the SMS rotates the carousel to place the oven beneath the knife-edge at the vacuum manifold interface to the mass spectrometer. Once in position, the carousel lifts the oven into the knife-edge (Fig. 7, right inset) and cleats on either side of the oven top flange preload the copper gasket on the oven flange to the 1055 steel knife-edge mounted to the manifold to create a leak tight seal.

Based on experience testing the SAM SMS, we decided to issue the same command, regardless of the number of seals that have been made with the oven. The nature of the steel knife-edge on the annealed soft copper gasket is such that if a sufficient amount of force is used, a seal can be made multiple times without the need for incrementing the force on successive seals. To date we have demonstrated over 20 leak tight seals with the SMS on a single VAPoR oven, each capable of holding a pressure of $\sim 10^{-8}$ mbar inside the oven with active pumping. Although dust contamination of the VAPoR could not be avoided in the field, the presence of dust on the oven and SMS surfaces did not affect the ability to obtain repeated leak tight seals. It should be noted that VAPoR operation on the Moon or another other airless body would not require a vacuum pump. However, a leak tight seal to the oven provided by an SMS would still be desirable in order to maintain gas pressure inside the mass spectrometer for static measurements of noble gases and other trace volatile components that would require much longer mass spectrometer integration times to increase signal to noise.

Finally, the SMS detach routine allows for easy insertion and removal of pyrolysis ovens without extensive disassembly of the SMS. All routines were tested during the DRATS campaign. The SMS successfully demonstrated robotic pyrolysis experiments in the field. Samples collected by DRATS crewmembers consisting of a team of astronauts and geologists were crushed and sieved by the VAPoR team. Approximately $10 \mathrm{mg}$ of sample powder was transferred to a quartz sample tube, the tube delivered to the pyrolysis oven through the SMS sample inlet location and subsequently sealed at the mass spectrometer interface, and the sample pyrolyzed for evolved gas measurements by the RGA. 


\section{EVOLVED GAS MEASUREMENTS AND FIELD RESULTS}

Evolved gas data provided by VAPoR can be used to select the most volatile-rich samples for large scale in situ resource utilization (ISRU) and/or sample return. To establish the volatile background inside the instrument, several procedural blank measurements of the fused silica and empty quartz sample tube and wool were made throughout the DRATS field test. Evolved gas analysis (EGA) data from a quartz tube procedural blank (Fig. 8) showed minor amounts $\left(<3 \times 10^{-7}\right.$ Torr $)$ of $\mathrm{H}_{2} \mathrm{O}(\mathrm{m} / \mathrm{z} 18), \mathrm{CO} / \mathrm{N}_{2}(\mathrm{~m} / \mathrm{z} 28)$, and $\mathrm{CO}_{2}$ $(\mathrm{m} / \mathrm{z} 44)$, with only traces $\left(<4 \times 10^{-9}\right.$ Torr $)$ of aliphatic $(\mathrm{m} / \mathrm{z}$ 27 and $\mathrm{m} / \mathrm{z} 30)$ and aromatic hydrocarbon fragments $(\mathrm{m} / \mathrm{z} 78)$, and $\mathrm{O}_{2}(\mathrm{~m} / \mathrm{z} 32)$. Other procedural blanks ran in the field yielded similar EGA results. The outgassing levels observed in the procedural blanks were much lower than the partial pressures of the same volatiles observed during pyrolysis of samples collected in the field $\left(\sim 10^{-5}\right.$ to $10^{-8}$ Torr range).

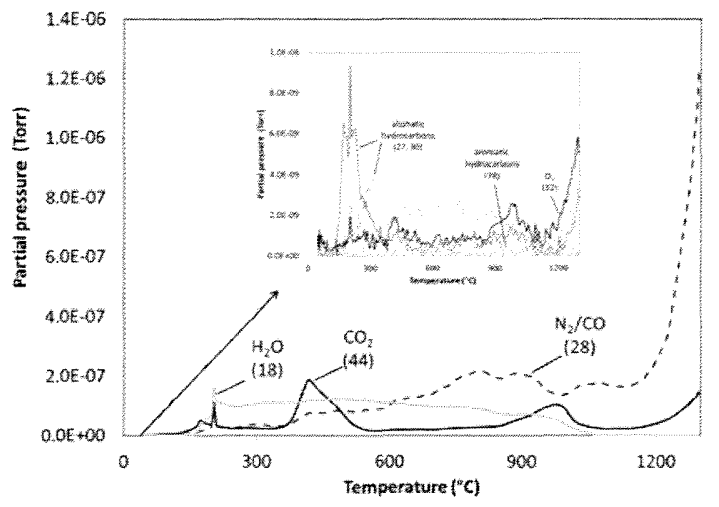

Fig. 8. Procedural blank background evolved gas profile of an empty quartz sample holder heated to $1300^{\circ} \mathrm{C}$. Selected inorganic and organic volatiles released from the sample as function of sample temperature. $\mathrm{N}_{2}$ and $\mathrm{CO}$ and cannot be separated with the RGA used and are therefore plotted together.

EGA data obtained from Geolab sample 0212, a vesicular basalt collected from the Black Point Lava Flow in Arizona is shown in Fig. 9. The primary volatile released between $300-$ $600^{\circ} \mathrm{C}$ was $\mathrm{CO}_{2}(\mathrm{~m} / \mathrm{z} 44)$ derived from the breakdown of carbonate minerals and possibly some oxidation of organic matter in the sample. Hydrocarbons were released from the sample at temperatures above $200^{\circ} \mathrm{C}$, consisting primarily of alkanes as indicated by characteristic alkane hydrocarbon fragment peaks at $\mathrm{m} / \mathrm{z} 27$ and $\mathrm{m} / \mathrm{z} 30$. Only trace levels of aromatic hydrocarbons $(\mathrm{m} / \mathrm{z} 78$, benzene) were detected in the sample. Some of these organics were released over the same temperature range as the carbonate mineral decomposition and therefore may have been trapped within the carbonate mineral matrix.

The water $(\mathrm{m} / \mathrm{z} \quad 18)$ peaks indicate the presence of multiple hydrated mineral phases, possibly hydrous oxides or phyllosilicates as well as hydrated sulfate minerals at trace levels, evidenced by the high-temperature $\mathrm{SO}_{2}(\mathrm{~m} / \mathrm{z}$ 64) peak seen at $\sim 960^{\circ} \mathrm{C} . \quad \mathrm{SO}_{2}$ release also occurred at the same temperature as carbonate decomposition, suggesting that a sulfate mineral was trapped in the carbonate mineral matrix. At temperatures of $\sim 350-600^{\circ} \mathrm{C}$, we observed a large $\mathrm{m} / \mathrm{z} 32$ peak, derived from a combination of ${ }^{32} \mathrm{~S}$ from $\mathrm{SO}_{2}$ and $\mathrm{O}_{2}$ released from multiple sources, including $\mathrm{CO}_{2}, \mathrm{SO}_{2}, \mathrm{H}_{2} \mathrm{O}$, and possibly oxides. The peaks at $\mathrm{m} / \mathrm{z} 28$ primarily indicate $\mathrm{CO}$ associated with degradation of carbonate minerals and oxidation of organic compounds, but a small percentage may represent $\mathrm{N}_{2}$. The high-temperature $\mathrm{m} / \mathrm{z} 28$ peak that is observed in both sample 0212 and the procedural blank is believed to derive from $\mathrm{N}_{2}$ and/or $\mathrm{CO}$ outgassing from the alumina crucible itself. To enable more accurate mineral assignments using this instrument in the future, an EGA library will be created from analysis data of a variety of pure mineral standards under the same pyrolysis conditions.

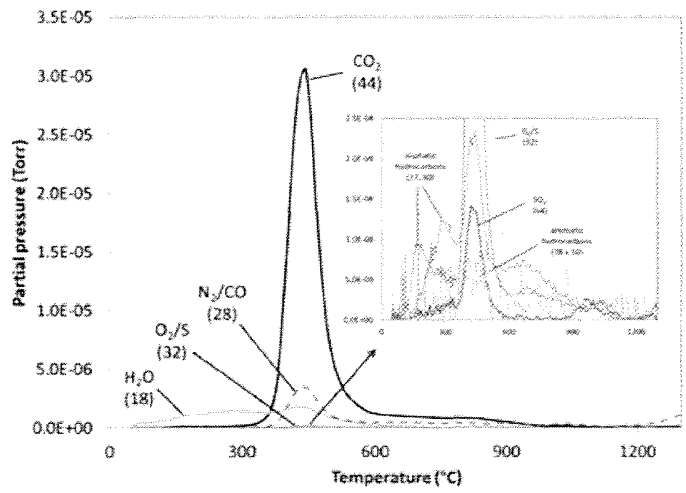

Fig. 9. VAPoR evolved gas analysis of DRATS sample 0212, a vesicular basalt collected at Black Point Lava Flow in Arizona, showing selected inorganic and organic volatiles released from the sample as a function of sample temperature.

\section{V.TIME OF FLIGHT MASS SPECTROMETER}

To identify trace volatiles of interest with similar masses (e.g. ${ }^{3} \mathrm{He} / \mathrm{HD}, \mathrm{N}_{2} \mathrm{H}_{4} / \mathrm{O}_{2}$ ) and make isotopic measurements, mass spectrometers with higher mass resolution and sensitivity than our current RGA quadrupole mass spectrometer will be required. We are currently developing a miniaturized reflectron time of flight mass spectrometer (TOF-MS) for VAPoR. The design of the TOF-MS component of VAPoR has been described previously [26], but the mass spectrometer has recently been modified with a new extended ion source and lens assembly needed to improve instrument sensitivity. The new TOF-MS has been systematically tested in both linear and reflectron modes (Fig. 10). In the reflectron (ion mirror) mode the direction of the ions is reversed in the reflectron and the ions are measured at a detector placed close to the ion source. The TOF-MS is mounted inside a vacuum chamber with a base pressure of $10^{-8}$ Torr. In parallel with the experimental testing, electrodynamics simulations of the TOFMS with the new extended ion source have been conducted using SIMION in both linear and reflectron modes.

The TOF-MS above consists of a carbon nanotube (CNT) field emission electron gun, $\mathrm{NiCr}$ ion extraction, steering, and focusing lenses, a monolithic curved-field reflectron, and two microchannel plate (MCP) detectors (one each for linear and reflectron modes) operated in ion counting mode (Fig. 10). During operation, ions are first generated by electron ionization within the ion source. High-speed electronics then pulse the ion lens voltages to accelerate ionized species into 
the TOF analyzer. The ions are then separated by mass and arrive as isomass packets at the detector.
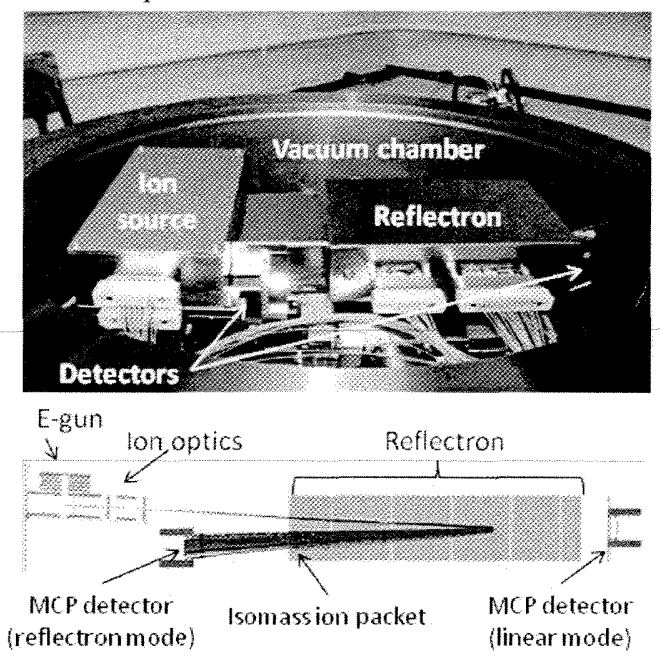

Fig. 10. The VAPOR TOF-MS shown inside a vacuum chamber at NASA Goddard can now be operated in both linear and reflectron modes. A new extended carbon nanotube electron gun (CNT e-gun) has recently been integrated to the TOF-MS for improved sensitivity over the previous small format e-gun.

By measuring MCP voltage as a function of time, a mass spectrum is acquired. The new extended ion source has a much larger ionization volume and ion transmission. Based on our calculations and SIMION modeling, we estimate that the new extended ion source will yield a sensitivity improvment of three orders of magnitude over previous reports using a smaller electron gun [26]. Details of the scaled-up electron gun design will be discussed elsewhere [Southard et al. in preparation].

The highest mass resolution performance is obtained in the reflectron mode of the TOF-MS. The effect of the reflectron is to lengthen the flight path for better temporal separation and better spatial focusing of the ion packets. In the current TOF-MS prototype, we conducted similar optimization efforts to that described in the linear case above, with the addition of tuning the curved field profile of the reflectron by adjusting individual voltages along the reflectron length. Experimentally, performance improvements have been demonstrated in mass separation and mass resolution, compared to the linear case above. For example, at mass 28 $\left(\mathrm{N}_{2} / \mathrm{CO}\right)$, the mass resolution $(\mathrm{m} / \Delta \mathrm{m})$ is 120 , nearly twice the mass resolution of the linear case which is $\sim 65$. We are continuing to optimize the tuning and alignment of the TOFMS instrument to improve performance and achieve the mass resolution and sensitivity requirements for the VAPoR instrument. Our ultimate target for the VAPoR TOF-MS is to achieve a mass resolution of $\sim 600-1000$ to enable separate of several key volatile species of interest on the lunar surface and improve the sensitivity of the mass spectrometer to $\sim 3 \times 10^{-4}$ counts $/ \mathrm{sec} / \mathrm{molecule} / \mathrm{cc}$. The addition of a time of flight mass spectrometer with enhanced sensitivity and improved mass resolution compared to the current RGA on a flight instrument should make it possible to identify some species (e.g., ${ }^{3} \mathrm{He} / \mathrm{HD}$ ) that we are currently unable to resolve with the VAPoR field unit.

\section{CONCLUSION}

In situ vacuum pyrolysis evolved gas measurements of the lunar regolith and other airless bodies including asteroids are needed to characterize and determine the origins of volatiles, particularly, water, an important resource for future human exploration. Using the VAPoR instrument during NASA's 2011 Desert RATS field campaign, we successfully demonstrated that high temperature vacuum pyrolysis of solid samples to temperatures exceeding $1300^{\circ} \mathrm{C}$ coupled with line of sight detection of volatiles by mass spectrometry can be used for the identification of resources including water and oxygen in surface samples. The inclusion of evolved gas analysis capability in the field and continued testing of instruments such as VAPoR in future field tests will be critical to the success of future robotic and human planetary resource exploration missions. The development of sample collection protocols designed to minimize or eliminate contamination from analyses such as those conducted by VAPoR are critical considerations for future space exploration architecture planning.

\section{ACKNOWLEDGMENTS}

The authors would like to acknowledge support from the NASA Astrobiology Science and Technology Instrument Development Program (07-ASTID07-0020), the NASA Moon and Mars Analog Mission Activities Program (10MMAMA10-0001), and the NASA Goddard Space Flight Center Internal Research and Development Program. We also thank B. A. Janoiko for logistical support in the field and $J$. Farmer and C. Evans for assistance with the sample collection and allocation protocol for VAPoR.

\section{REFERENCES}

[1] A. Colaprete, et al., "Detection of Water in the LCROSS Ejecta Plume," Science, vol. 330, pp. 463-468, Oct 222010.

[2] D. B. Bussey, et al., "Planetary science: constant illumination at the lunar north pole," Nature, vol. 434, p. 842, 2005.

[3] B. A. Cohen, et al., "Support for the lunar cataclysm hypothesis from hunar meteorite impact melt ages," Science, vol. 290, pp. 1754-1756, Dec 12000

[4] D. H. Crider and R. R. Vondrak, "Hydrogen migration to the lunar poles by solar wind bombardment of the moon," Lunar Exploration 2000, vol. 30, pp. 1869-1874, 2002.

[5] G. De Maria, et al., "Mass spectrometric investigation of the vaporization process of Apollo 12 lunar samples," Proceedings of the Second Lunar Science Conference, vol. 2, pp. 1367-1380, 1971.

[6] E. K. Gibson Jr. and S. M. Johnson, "Thermal analysis - inorganic gas release studies of lunar samples, "Proceedings of the Second Lunar Science Conference, vol. 2, pp. 1351-1366, 1971.

[7] B. R. Simoneit, et al., "Volatile element chemistry of selected lunar, meteoritic, and terrestrial samples," Proceedings of the Fourth Lunar Science Conference, vol. 4, pp. 1635-1650, 1973.

[8] A. E. Saal, et al., "Volatile content of lunar volcanic glasses and the presence of water in the Moon's interior," Nature, vol. 454, pp. 192-U38, Jul 102008.

[9] J. H. Hoffman, et al., "Lunar Atmosphere - Results from Apollo17," Transactions-American Geophysical Union, vol. 54, pp. $387-$ $387,1973$.

[10] D. M. Anderson, et al., "Mass-Spectrometric Analysis of Organic Compounds, Water and Volatile Constituents in Atmosphere and Surface of Mars - Viking Mars Lander," Icarus, vol. 16, pp. 111$\&, 1972$. 
[11] J. Matchett, et al., "A demonstration of vacuum pyrolysis," presented at the Space Resources Roundtable VII, Houston, TX, 2005.

[12] B. D. Lightner and K. Marti, "Lunar trapped xenon," presented at the Proceedings of the Fifth Lunar Science Conference, 1974.

[13] D. Bogard, D. and E. K. Gibson Jr., "Volatile Gases in Breccia 68115," presented at the Proceedings of the Sixth Lunar Science Conference, 1975.

[14] E. K. Gibson Jr. and F. Andrawes, "Nature of the gas phases released from lunar rocks and soils upon crushing," presented at the Lunar and Planetary Science Conference, Houston, TX, 1978.

[15] K. Kehm, et al., "Combined noble gas and trace element measurements on individual stratospheric interplanetary dust particles," Meteoritics \& Planetary Science, vol. 37, pp. 13231335, Oct 2002.

[16] I. L. ten Kate, et al., "VAPoR - Volatile Analysis by Pyrolysis of Regolith - an instrument for in situ detection of water, noble gases, and organics on the Moon," Planetary and Space Science, vol. 58, pp. 1007-1017, Jun 2010.

[17] P. T. Holland, et al., "Compounds of carbon and other volatile elements in Apollo 14 and 15 samples," Proceedings of the Third Lunar Science Conference, vol. 3, pp. 2131-2147, 1972.

[18] R. W. Court, et al., "The combustion characteristics and stable carbon isotopic compositions of irradiated organic matter: Implications for terrestrial and extraterrestrial sample analysis," presented at the Lunar and Planetary Science Conference XXXVI, Houston, TX, 2005.

[19] A. E. Lord, "Hydrogen and helium ion implantation into olivine and enstatite: Retention coefficients, saturation concentrations, and temperature release profiles," Journal of Geophysical Research, vol. 73 , pp. 5271-5280, 1968.

[20] D. M. Anderson, et al., "Mass-Spectrometric Analysis of Organic Compounds, Water and Volatile Constituents in Atmosphere and Surface of Mars - Viking Mars Lander," Icarus, vol. 16, pp. 111138, 1972.

[21] K. Biemann, et al., "The search for organic substances and inorganic volatile compounds in the surface of Mars," Journal of Geophysical Research, vol. 82, pp. 4641-4658, 1977.

[22] W. V. Boynton, et al., "Thermal and Evolved Gas Analyzer: Part of the Mars Volatile and Climate Surveyor integrated payload," Journal of Geophysical Research-Planets, vol. 106, pp. 1768317698, Aug 252001.

[23] F. Goesmann, et al., "COSAC, the cometary sampling and composition experiment on Philae," Space Science Reviews, vol. 128 , pp. 257-280, 2007.

[24] I. P. Wright, et al., "Ptolemy - An instrument to measure stable isotopic ratios of key volatiles on a cometary nucleus," Space Science Reviews, vol. 128, pp. 363-381, 2007.

[25] P. R. Mahaffy, "Exploration of the habitability of Mars: Development of analytical protocols for measurement of organic carbon on the 2009 Mars Science Laboratory," Space Science Reviews, vol. 135, pp. 255-268, 2008.

[26] S. A. Getty, et al., "Development of an evolved gas-time-of-flight mass spectrometer for the Volatile Analysis by Pyrolysis of Regolith (VAPoR) instrument," International Journal of Mass Spectrometry, vol. 295, pp. 124-132, Aug 12010.

[27] D. D. Bogard, "K-Ar dating of rocks on Mars: Requirements from Martian meteorite analyses and isochron modeling," Meteoritics \& Planetary Science, vol. 44, pp. 3-14, Jan 2009.

[28] J. M. J. Hurtado, et al., "Field geologic observation and sample collection strategies for planetary surface exploration: Insights from the 2010 Desert RATS geologist crewmembers," Acta Astronautica, vol. in press, 2011.

[29] C. A. Evans, et al., "GeoLab - A habitat-based laboratory for preliminary examination of geological samples," Acta Astronautica, vol. in revision, 2011. 